\title{
Prevalence and Associations of Vitreomacular Traction: The Beijing Eye Study
}

\section{Lei Shao \\ Chuan Zhang \\ Li Dong \\ Wen Da Zhou \\ Rui Heng Zhang \\ Wen Bin Wei}

Beijing Tongren Eye Center, Beijing Key Laboratory of Intraocular Tumor Diagnosis and Treatment, Beijing Ophthalmology \& Visual Sciences Key Lab, Medical Artificial Intelligence Research and Verification Key Laboratory of the Ministry of Industry and Information Technology, Beijing Tongren Hospital, Capital Medical University, Beijing, People's Republic of China
Correspondence: Wen Bin Wei Beijing Tongren Eye Center, Beijing Tongren Hospital, Capital Medical University, I Dong Jiao Min Xiang, Dong Cheng District, Beijing, I00730, People's Republic of China

Tel +86-10-582695I6

Fax +86-10-6512 5617

Email weiwenbintr@163.com
Purpose: To explore the prevalence and associations of vitreous macular traction (VMT) in Chinese population.

Methods: A population-based cross-sectional study with 3468 individuals (mean age of $64.6 \pm 9.8$ years) based on Beijing Eye Study 2011. Participants underwent detailed ophthalmic examinations, including spectral domain optical coherence tomography (SD-OCT). VMT was defined as abnormal posterior vitreous detachment with anatomical deformation of the fovea, which may include pseudocyst, macular schisis, cystoid macular edema, and subretinal fluid.

Main Outcome Measure: Prevalence of VMT.

Results: A VMT was detected in $151(2.3 \%)$ eyes (prevalence rate (mean $\pm \mathrm{SE}$ ): $2.3 \pm 0.2 \%$; 95\% CI: $1.9 \%, 2.7 \%$ ) of $80(2.4 \%)$ subjects (prevalence rate: $2.4 \pm 0.3 \%$; $95 \%$ CI: $1.9 \%$, $2.9 \%$ ). Mean age of all subjects with VMT was $70.1 \pm 8.7$ years (median, 70.0 years; range, $50-90$ years), mean refractive error was $-0.22 \pm 2.38 \mathrm{D}$ (median, $0.25 \mathrm{D}$; range, -15.00 to 5.25D). In multivariate analysis, prevalence of VMT was associated with elder age $(\mathrm{P}=0.001$, OR 1.06), female gender $(\mathrm{P}=0.036$, OR 1.77), subfoveal retinal thickness $(\mathrm{P}=0.005, \mathrm{OR}$ $1.01)$, and subfoveal choroidal thickness $(\mathrm{P}=0.026$, OR 0.10).

Conclusion: In adult Chinese in Greater Beijing, the prevalence of VMT was $2.3 \%$ for eyes or $2.4 \%$ for subjects. While it was associated with elder age, female gender, thicker subfoveal retinal thickness and thinner subfoveal choroidal thickness.

Keywords: vitreous macular traction, optical coherence tomography, the Beijing Eye Study

\section{Introduction}

Vitreomacular traction syndrome (VMT) was assumed to be an uncommon entity and associated with other macular disorders. ${ }^{1}$ VMT is considered to be associated with a broad spectrum of macular diseases, including cystoid macular edema $(\mathrm{CME})$, epiretinal membrane (ERM) and macular hole $(\mathrm{MH})$, all of which are attributed to common etiology. ${ }^{2-5}$ The hallmark of VMT is a persistent attachment of the vitreous to the macula in eyes with an incomplete posterior vitreous detachment. In the most common form of VMT syndrome, as initially described, ${ }^{6}$ the vitreous is separated from the retina throughout the peripheral fundus but remains adherent posteriorly, producing anteroposterior traction on a narrow region of several optic disc areas, usually dumbbell-shaped areas, encompassing macular areas and optic nerves. ${ }^{2,7,8}$ Traction on the retina at the macula causes decreased vision, metamorphopsia, photopsia, and micropsia. ${ }^{2-5,7-10}$ Among vitreoretinal interface abnormalities, VMT syndrome is probably one of the conditions which has been significantly improved in terms of pathophysiologic concepts. 
Due to the limitation of three-dimensional optical resolution of ophthalmoscope and fundus photography, VMT could not reliably be diagnosed. However, spectral domain high-resolution OCT (HD-OCT) has provided new insights for the understanding of VMT syndrome by better evaluating the traction of vitreoretinal interface and understanding its relationship with specific macular conditions. ${ }^{3,9,10}$ Despite their clinical significance, besides a series of hospital-based studies, there has been no population-based investigation on the occurrence and associations of VMT with other ocular and general parameters. Therefore, we conducted this study to evaluate the prevalence of VMT and its related factors in population-based investigation.

\section{Methods}

The Beijing Eye Study 2011 is a population-based crosssectional study. The total population aged over 50 in the eight communities involved was 4403, of which 3468 (1963 (56.6\%) women) participated in ophthalmic examination, and the overall response rate was $78.8 \%$. The mean age of 3468 individuals was $64.6 \pm 9.8$ years (median, 64 years; range, 50-93 years). The study was divided into the rural part (1633 (47.1\%) subjects) and the urban part (1835 (52.9\%) subjects). According to the declaration of Helsinki, the Medical Ethics Committee of Beijing Tongren Hospital approved the research protocol, and all participants gave informed written consent.

All examinations were conducted in the community. By asking questions, the researchers extracted the subjects' demographic variables, socio-economic background and systemic diseases. Blood pressure, height, weight, waist circumference and hip circumference were measured. Fasting blood samples were collected to measure low-density lipoprotein, high-density lipoprotein, cholesterol, creatinine, triglyceride and blood glucose. All subjects underwent a comprehensive ophthalmic examination. Visual acuity (VA), best corrected visual acuity (BCVA, Nidek Co., Ltd., Tokyo, Japan), intraocular pressure (IOP, Goldmann tonometer) and slit lamp examination were recorded. The anterior segment examination (Haag-Streit, CH- 3098 Koeniz, Switzerland) and fundus examination (Optovue Inc. Fremont, CA, USA; Heidelberg Engineering, Heidelberg, Germany) were performed.

The patient underwent macular scanning through spectral domain optical coherence tomography (SD-OCT; Spectralis $^{\circledR}$, Heidelberg Engineering Co., Heidelberg, Germany) The examination included 100 average scans, centered on the fovea and scanned at a rectangular angle of $5^{\circ}-30^{\circ}$, a total of 7 times. In addition, the thickness of foveal choroid was measured by enhanced depth imaging modality. Heidelberg eye Explorer software (version 5.3.3.0; Heidelberg engineering company, Germany) was used for data measurement.

Only those subjects with macular OCT images were included. The OCT criteria (B-mode scan) that classify the eyes as VMT include: (1) perifoveal vitreous cortical detachment from the retinal surface; (2) the vitreous cortex of macula was attached within $3 \mathrm{~mm}$ of the radius of fovea; (3) association of attachment with distortion of the foveal surface, intraretinal structural changes, elevation of the fovea above the RPE, or a combination of no fullthickness interruption of all retinal layers The method of defining VMT is to use a photographic standard, as proposed by Simpson et al, ${ }^{11}$ and then described by the International Vitreomacular Traction Study Group in $2013 .^{7}$ Vitreomacular traction is by definition a structural abnormality, which causes focal, tractional, distortion of the macula. (Figure 1) The images were taken by one technician (CXC) and the images were assessed by an experienced and trained ophthalmologist (LS). In case of doubt, the OCT images were reassessed by a panel including several ophthalmologists (LS, WBW).

The statistical analysis was performed using a commercially available statistical software package (SPSS for Windows, version 25.0, SPSS, Chicago, IL, USA). The prevalence of a VMT was calculated. And we performed both univariate and multivariate logistic regression analyses to assess associations with VMT. Ninetyfive percent Confidence intervals (CI) were presented. All

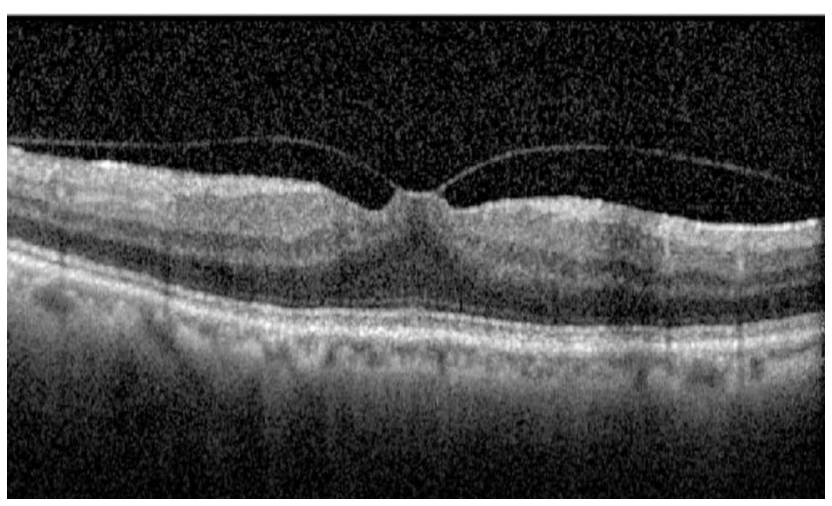

Figure I Optical coherence tomography of vitreomacular traction. That is by definition a structural abnormality, which causes focal, tractional, distortion of the macula. 
P-values were 2-sided and were considered statistically significant when $\mathrm{p}<0.05$.

\section{Results}

Of the 3468 participants included in the study, 6530 eyes in 3276 (94.5\%) subjects obtained sufficient quality OCT images for examination, while 192 (5.5\%) subjects were excluded. The main reasons for exclusion were available images could not be evaluated owing to refractive interstitial opacity, or images were not taken. The mean age of the included subjects was $64.3 \pm 9.6$ years (50 to 93 years), and the mean ametropia (spherical equivalent) was $-0.18 \pm 2.04$ diopter (- 22.0 to +7.50 diopter). The age of subjects who did not receive OCT increased significantly compared with those who received OCT $(70.1 \pm 11.2$ years, $64.3 \pm 9.6$ years; $\mathrm{P}<$ 0.05 ; $95 \% \mathrm{CI}: 4.15,7.39)$, but there was no significant difference in gender $(\mathrm{P}=0.12)$ and ametropia $(\mathrm{P}=0.29)$.

The prevalence of VMT was $2.3 \% \pm 0.2 \%$ for eyes $(151$ eyes, $95 \%$ CI: $1.9-2.7 \%$ ) or $2.4 \% \pm 0.3 \%$ for subjects $(80$ subjects, 95\% CI: 1.9-2.9\%). Mean age of all subjects with VMT was $70.1 \pm 8.7$ years (median: 70.0 years; range: 50 to 90 years), mean refractive error was $-0.22 \pm$ 2.38D (median, $0.25 \mathrm{D}$; range, -15.00 to $5.25 \mathrm{D}$ ).

If in a bivariate analysis the subjects with VMTs were compared with the subjects without VMTs, the prevalence of VMT was significantly associated with the systemic parameters of elder age $(\mathrm{P}<0.001)$ (Figure 2$)$, female gender $(\mathrm{P}=$ $0.046)$, and lower alcohol consumption $(\mathrm{P}=0.039)$, and with

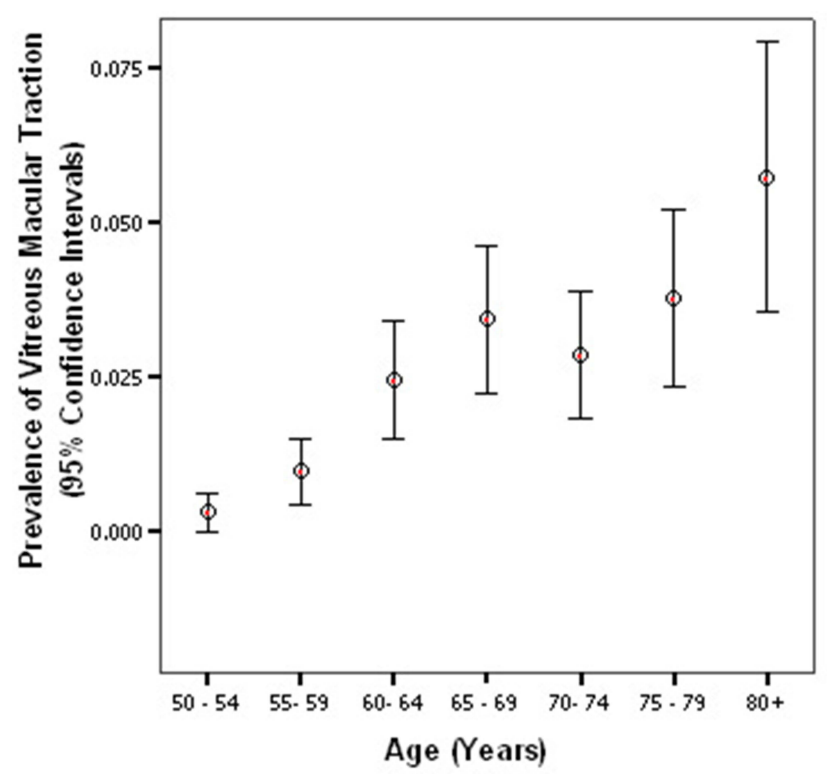

Figure 2 Diagram showing the distribution of prevalence of vitreous macular traction stratified by age groups in Beijing Eye Study 2011. the ocular parameters of thicker lens $(\mathrm{P}=0.026)$, lower bestcorrected visual acuity (LogMAR) $(\mathrm{P}=0.027)$, thicker subfoveal retinal thickness $(\mathrm{P}<0.001)$ and thinner choroidal thickness $(\mathrm{P}<0.001)$, small pupil diameter $(\mathrm{P}=0.035)$. It was not significantly associated with the systemic parameters of body height ( $\mathrm{P}=0.142)$, weight $(\mathrm{P}=0.441)$, cognitive level $(\mathrm{P}=0.369)$, systolic blood pressure $(\mathrm{P}=0.948)$, diastolic blood pressure $(\mathrm{P}=0.371)$, serum concentrations of high-density lipoproteins ( $\mathrm{P}=0.800)$, low-density lipoproteins $(\mathrm{P}=0.819)$, cholesterol $(\mathrm{P}=0.822)$, creatine $(\mathrm{P}=$ $0.582)$, triglycerides $(P=0.625)$ and glucose $(P=0.302)$, history of hypertension $(\mathrm{P}=0.804)$, hyperlipidemia $(\mathrm{P}=$ 0.997), diabetes mellitus ( $\mathrm{P}=0.256)$, self-reported diagnosis of cerebral infarction or haemorrhage $(\mathrm{P}=0.478)$, and of coronary heart disease $(\mathrm{P}=0.083)$, frequency of reported snoring $(\mathrm{P}=0.094)$, smoking $(\mathrm{P}=0.163)$, and rural region of habitation $(\mathrm{P}=0.145)$; and with the ocular parameters of axial length $(P=0.856)$, refractive error $(P=0.784)$, intraocular pressure $(\mathrm{P}=0.262)$, anterior chamber depth $(\mathrm{P}=$ $0.395)$, central corneal thickness $(P=0.990)$, corneal diameter $(\mathrm{P}=0.548)$, corneal steep $(\mathrm{P}=0.912)$, and history of cataract surgery $(\mathrm{P}=0.300)$ (Table 1$)$.

In a multivariate logistic regression analysis (backward stepwise method), the prevalence of VMT was no longer significantly associated with parameters of best corrected visual acuity $(\mathrm{P}=0.059)$, lens thickness $(\mathrm{P}=0.956)$, pupil diameter $(\mathrm{P}=0.093)$, and alcohol consumption $(\mathrm{P}=$ 0.151). The associations between the presence of VMT and elder age $(\mathrm{P}=0.001, \mathrm{OR} 1.06)$, female gender $(\mathrm{P}=$ 0.036, OR 1.77), subfoveal retinal thickness $(\mathrm{P}=0.005$, OR 1.01), subfoveal choroidal thickness $(\mathrm{P}=0.026$, OR 0.10 ) remained statistically significant (Table 2 ).

\section{Discussion}

In our population-based study on adult Chinese in Beijing, the prevalence of VMT was $2.3 \pm 0.2 \%$ per eye or $2.4 \pm$ $0.3 \%$ per subject. Moreover, it was associated with elder age $(\mathrm{P}=0.001)$, female gender $(\mathrm{P}=0.036)$, subfoveal retinal thickness $(\mathrm{P}=0.005)$, subfoveal choroidal thickness $(\mathrm{P}=0.026)$.

These results can not be compared with any other population-based investigations, resulting from that only few population-based studies have applied OCT technology for imaging of the posterior ocular segment, such as the Handan Study, Sydney Myopia Study, and Singapore Chinese Eye Study. But none of these studies examined the presence of a VMT. ${ }^{12-14}$ Recent, Menzler et $\mathrm{al}^{15}$ has reported the prevalence of symptomatic vitreomacular adhesion, 
Table I Factors Associated with Vitreous Macular Traction in the Beijing Eye Study (Univariate Analysis)

\begin{tabular}{|l|c|c|c|}
\hline Factor & P-value & OR & $\begin{array}{c}\text { 95\% Confidence } \\
\text { Interval }\end{array}$ \\
\hline Gender & 0.046 & I.4I & I.0I, I.97 \\
Age & $<0.001$ & 1.07 & $1.05,1.09$ \\
Region of habitation & 0.145 & & \\
Body height & 0.142 & & \\
Body weight & 0.441 & & \\
Cognitive level & 0.369 & & \\
Diastolic blood pressure & 0.371 & & \\
Systolic blood pressure & 0.948 & & \\
Low-density lipoproteins & 0.819 & & \\
High-density lipoproteins & 0.800 & & \\
Cholesterol & 0.822 & & \\
Creatinine & 0.582 & & \\
Triglycerides & 0.625 & & \\
Glucose & 0.302 & & \\
Known diabetes mellitus & 0.256 & & \\
Known coronary heart disease & 0.083 & & \\
Known Hypertension & 0.804 & & \\
Known hyperlipidemia & 0.997 & & \\
Cerebral infarction or haemorrhage & 0.478 & & \\
Smoking & 0.163 & & \\
Alcohol consumption & 0.039 & 0.84 & $0.71,0.99,1.00,1.01$ \\
Snoring & 0.094 & & \\
Cataract surgery & 0.300 & & \\
Axial length & 0.856 & & \\
Refractive error & 0.784 & & \\
Anterior chamber depth & 0.395 & & \\
Lens thickness & 0.026 & 2.21 & \\
Central corneal thickness & 0.990 & & \\
Corneal diameter & 0.548 & & \\
Corneal steep & 0.912 & & \\
Pupil diameter & 0.035 & 0.70 & \\
Best corrected visual acuity (LogMAR) & 0.027 & 0.36 & \\
Intraocular pressure & 0.262 & & \\
Subfoveal choroidal thickness & $<.001$ & 0.10 & \\
Subfoveal retinal thickness & $<.001$ & 1.01 & \\
\hline
\end{tabular}

which is 1365 per 100,000 population. Symptomatic vitreomacular adhesion can occur in isolation as VMT, which has the symptoms of decreased visual acuity, metamorphopsia,

Table 2 Factors Associated with Vitreous Macular Traction Using Multivariate Logistic Regression Models in the Beijing Eye Study

\begin{tabular}{|l|c|c|c|}
\hline Factor & P-value & OR & 95\% Confidence Interval \\
\hline Gender & 0.036 & 1.77 & $1.04,3.02$ \\
Age & 0.001 & 1.06 & $1.03,1.10$ \\
Subfoveal choroidal thickness & 0.026 & 0.10 & $0.99,1.00$ \\
Subfoveal retinal thickness & 0.005 & 1.01 & $1.00,1.01$ \\
\hline
\end{tabular}

micropsia, and even photopsia. Furthermore, it may lead to the development of a macular hole, or it may occur alongside an epiretinal membrane. It is likely to be associated with agerelated macular degeneration and possibly diabetic maculopathy, although this is less certain. In our study, the results involved subjects both of nonsymptomatic vitreomacular traction and symptomatic vitreomacular adhesion. If we take the figures of Menzler $\mathbf{J}$ as a complement to our study, we may consider that about $1 \%$ of subjects could have classic VMT symptoms and high risks in associated diseases.

Interestingly, several clinical-based studies revealed that the occurrence of a VMT was significantly associated with elder age and the mean age of patients diagnosed with VMT is around 65-75 years (range 48-83 years), with a predominance of females. ${ }^{7,16-18}$ These findings confirm our results. VMT is a special stage of posterior vitreous detachment (PVD), and studies in healthy adults with otherwise normal eyes have shown that posterior vitreous detachment (PVD) occurs in around 50\% at the ages 30 39 years, whereas complete PVD is observed in $50 \%$ or more of individuals more than aged 70 years. ${ }^{19,20}$ Therefore, the prevalence of VMT was also higher in elder population and associated with age. Moreover, VMT is significantly more common in postmenopausal women than men; this may be due to the effects of decreased oestrogen on connective tissues such as those within the vitreous gel. ${ }^{21,22}$

In our population-based study, VMT also correlated to the thicker retina and thinner choroid. The associations between VMT and subfoveal thickness of retina or choroid have remained unclear so far. One of the reasons may be the traction between posterior vitreous cortex and internal limiting lamina of retina, which stretched the layers of retina when VMT occurs. If the structure of retinal layers was elongated, the thickness of retina could increase when measured by OCT. Furthermore, thinner choroidal thickness in VMTs may correlate to the decreased local blood circulation of choroids.

The associations between the VMT and parameters of best corrected visual acuity, lens thickness, pupil diameter, alcohol consumption as found in bivariate analysis were no longer statistically significant in the multivariate analysis after adjusting for age, gender subfoveal retinal thickness and subfoveal choroidal thickness. It showed that the associations between the presence of VMT and best corrected visual acuity, lens thickness, pupil diameter, alcohol consumption were merely explained by the confounding effects of the correlations of age, gender subfoveal retinal thickness and subfoveal choroidal thickness. 
The findings in our study may have clinical importance, since VMT may associate with several pathological processes of posterior poles, such as $\mathrm{MH}, \mathrm{CME}, \mathrm{ERM},{ }^{2-5}$ and even agerelated macular degeneration. ${ }^{5,10,11}$ Further studies may assess whether the results of our study help in understanding the pathogenesis of these disorders by showing the correlations of VMT and other ocular and general parameters. If that is the case, one may hypothetically consider that releasing the vitreomacular traction artificially either by operation or drugs, ${ }^{23,24}$ such as ocriplasmin, may control or cure the associated diseases.

Potential limitations of our study should be mentioned. First, a major concern in any prevalence study is nonparticipation. The Beijing Eye Study 2011 had a reasonable response rate of $78.8 \%$, however, differences between participants and non-participants could have led to a selection artifact. Since the group of subjects without OCT examinations as compared with the group of subjects with OCT examinations was significantly $(\mathrm{P}<0.001)$ older, one may argue that the non-participation of a part of the elderly eligible study population may have influenced the results of the investigation. Second, although examinations of VMT by a group of trained ophthalmologists, the photographic standard to define VMT may introduce a bias into our study. Third, the OCT method may not have imaged all VMTs, especially tractions located in periphery region. The strengths of our study are it is the first population-based investigation searching for the prevalence and associations between ocular and systemic parameters and VMT in adult Chinese.

In conclusion, in adult Chinese in Beijing, the prevalence of VMT was $2.3 \%$ for eyes or $2.4 \%$ for subjects. While it was associated with elder age, female gender, thicker subfoveal retinal thickness and thinner subfoveal choroidal thickness.

\section{Data Sharing Statement}

The raw data supporting the conclusions of this article will be made available by the correspondence authors to any qualified researcher with appropriate requests.

\section{Author Contributions}

Design of the study: Shao L, Wei WB; development of the algorithm: Shao L; gathering the data: Shao L, Zhang C, Dong L, Zhou WD, Zhang RH; performing the data analysis: Shao L; drafting the first version of the manuscript: Shao L, Zhang C. All authors made a significant contribution to the work reported, whether that is in the conception, study design, execution, acquisition of data, analysis and interpretation, or in all these areas; took part in drafting, revising or critically reviewing the article; gave final approval of the version to be published; have agreed on the journal to which the article has been submitted; and agree to be accountable for all aspects of the work.

\section{Funding}

This study was supported by National Natural Science Foundation of China (No. 82000916). The priming scientific research foundation for the junior researcher in Beijing Tongren Hospital, Capital Medical University (2016-YJJ-ZLL-009); Beijing Hospitals Authority Youth Programme, code: QML20180204; The priming scientific research foundation for the junior researcher in Beijing Tongren Hospital, Capital Medical University (No.2018YJJ-ZZL-045). Dongcheng District Outstanding Talent Nurturing Program (2020-dchrcpyzz-42).

\section{Disclosure}

The authors declare that the research was conducted in the absence of any commercial or financial relationships that could be construed as a potential conflict of interest.

\section{References}

1. Shao L, Wei W. Vitreomacular traction syndrome. Chin Med J. 2014;127:1566-1571.

2. Carpineto P, Ciciarelli V, Borrelli E, Aharrh-Gnama A, Mastropasqua R. Epiretinal membrane in eyes with vitreomacular traction. Retina. 2019;39:1061-1065. doi:10.1097/IAE.0000000000002546

3. Okada M, Chiu D, Yeoh J. Vitreomacular disorders: a review of the classification, pathogenesis and treatment paradigms including new surgical techniques. Clin Exp Optom. 2021;1-12. doi:10.1080/ 08164622.2021 .1896946

4. Schumann RG, Herold TR, Haritoglou C, Maier M, Lommatzsch A. Retinal imaging of vitreomacular traction diseases. Ophthalmologe. 2021;118:291-302. doi:10.1007/s00347-020-01309-w

5. Wang Y, Zhao X, Zhang W, Yang J, Chen Y. Fovea-sparing versus complete internal limiting membrane peeling in vitrectomy for vitreomacular interface diseases: a systematic review and meta-analysis. Retina. 2021;41:1143-1152.

6. Reese AB, Jones IS, Cooper WC. Vitreomacular traction syndrome confirmed histologically. Am J Ophthalmol. 1970;69:975-977. doi:10.1016/0002-9394(70)91041-X

7. Duker JS, Kaiser PK, Binder S, et al. The International Vitreomacular Traction Study Group classification of vitreomacular adhesion, traction, and macular hole. Ophthalmology. 2013;120:2611-2619. doi:10.1016/j.ophtha.2013.07.042

8. Tsokolas G, Tsaousis KT, Diakonis VF, Tyradellis S, Konidaris V. Spontaneous resolution of vitreomacular traction. Clin Exp Optom. 2020;103:386-389. doi:10.1111/cxo.12970

9. Chan CK, Mein CE, Glassman AR, et al. Pneumatic vitreolysis with perfluoropropane for vitreomacular traction with and without macular hole: DRCR Retina Network Protocols AG and AH. Ophthalmology. 2021. doi:10.1016/j.ophtha.2021.05.005

10. Haritoglou C, Hubschman JP, Schumann RG, Maier M. Optical coherence tomography biomarkers in epimacular membranes and vitreomacular traction syndrome. Ophthalmologe. 2021;118:308-319. doi:10.1007/s00347-021-01349-w 
11. Simpson AR, Petrarca R, Jackson TL. Vitreomacular adhesion and neovascular age-related macular degeneration. Surv Ophthalmol. 2012;57:498-509. doi:10.1016/j.survophthal.2012.01.011

12. Hu A, Gu SZ, Friedman DS, Cao K, Wang N. Six-year incidence and causes of low vision and blindness in a rural Chinese adult population: the Handan Eye Study. Ophthalmic Epidemiol. 2021;28:160-168. doi:10.1080/09286586.2020.1795886

13. Kidd Man RE, Liang Gan AT, Fenwick EK, et al. Using uniocular visual acuity substantially underestimates the impact of visual impairment on quality of life compared with binocular visual acuity. Ophthalmology. 2020;127:1145-1151. doi:10.1016/j.ophtha.20 20.01.056

14. Philip K, Sankaridurg PR, Ale JB, Naduvilath TJ, Mitchell P. Profile of off-axis higher order aberrations and its variation with time among various refractive error groups. Vision Res. 2018;153:111-123. doi:10.1016/j.visres.2018.07.005

15. Menzler J, Neubauer AS, Haritoglou C, Jackson TL. Incidence and prevalence of vitreomacular traction with and without macular hole in Germany. Clin Ophthalmol. 2019;28:177-188. doi:10.2147/OPTH. S188704

16. Flaxel CJ, Adelman RA, Bailey ST, et al. Idiopathic epiretinal membrane and vitreomacular traction preferred practice Pattern ${ }^{\mathbb{}}$. Ophthalmology. 2020;127:P145-p183. doi:10.1016/j.ophtha.20 19.09 .022

17. Fukumoto M, Sato T, Oosuka S, Kida T, Oku H, Ikeda T. Clinical features of vitreomacular traction syndrome with peripheral vitreoretinal adhesion. Clin Ophthalmol. 2020;14:281-286.
18. Petrou P, Chalkiadaki E, Errera MH, et al. Factors associated with the clinical course of vitreomacular traction. $J$ Ophthalmol. 2020;2020:9457670.

19. Shao L, Xu L, You QS, et al. Prevalence and associations of incomplete posterior vitreous detachment in adult Chinese: the Beijing Eye Study. PLoS One. 2013;8:e58498. doi:10.1371/journal.pone.0058498

20. Uchino E, Uemura A, Ohba N. Initial stages of posterior vitreous detachment in healthy eyes of older persons evaluated by optical coherence tomography. Arch Ophthalmol. 2001;119:1475-1479. doi:10.1001/archopht.119.10.1475

21. Chuo JY, Lee TY, Hollands $\mathrm{H}$, et al. Risk factors for posterior vitreous detachment: a case-control study. Am J Ophthalmol. 2006;142:931-937. doi:10.1016/j.ajo.2006.08.002

22. Hwang ES, Kraker JA, Griffin KJ, Sebag J, Weinberg DV, Kim JE. Accuracy of spectral-domain OCT of the macula for detection of complete posterior vitreous detachment. Ophthalmol Retina. 2020;4:148-153. doi:10.1016/j.oret.2019.10.013

23. Stalmans P, Benz MS, Gandorfer A, et al. Enzymatic vitreolysis with ocriplasmin for vitreomacular traction and macular holes. $N$ Engl $J$ Med. 2012;367:606-615. doi:10.1056/NEJMoa1110823

24. Steel DHW, Patton N, Stappler T, et al. Ocriplasmin for vitreomacular traction in clinical practice: the INJECT Study. Retina. 2021;41:266-276. doi:10.1097/IAE.0000000000002862
International Journal of General Medicine

\section{Publish your work in this journal}

The International Journal of General Medicine is an international, peer-reviewed open-access journal that focuses on general and internal medicine, pathogenesis, epidemiology, diagnosis, monitoring and treatment protocols. The journal is characterized by the rapid reporting of reviews, original research and clinical studies

\section{Dovepress}

across all disease areas. The manuscript management system is completely online and includes a very quick and fair peer-review system, which is all easy to use. Visit http://www.dovepress.com/ testimonials.php to read real quotes from published authors. 